\title{
Lipschitz-free Banach spaces
}

\author{
by \\ G. Godefroy (Paris) and N. J. Kalton (Columbia, MO)
}

\begin{abstract}
We show that when a linear quotient map to a separable Banach space $X$ has a Lipschitz right inverse, then it has a linear right inverse. If a separable space $X$ embeds isometrically into a Banach space $Y$, then $Y$ contains an isometric linear copy of $X$. This is false for every nonseparable weakly compactly generated Banach space $X$. Canonical examples of nonseparable Banach spaces which are Lipschitz isomorphic but not linearly isomorphic are constructed. If a Banach space $X$ has the bounded approximation property and $Y$ is Lipschitz isomorphic to $X$, then $Y$ has the bounded approximation property.
\end{abstract}

1. Introduction. A (real) Banach space $X$ is in particular a metric space, equipped with the distinguished point $\{0\}$, and to such a pointed

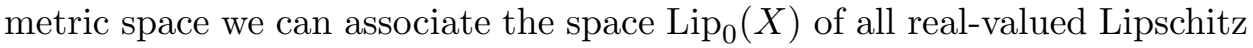
functions which vanish at 0 . We refer to [29] for basic facts on this space and some of its uses. It is clear that $\operatorname{Lip}_{0}(X)$ is a Banach space when it is equipped with the Lipschitz norm, defined by

$$
\|f\|_{\text {Lip }}=\sup \left\{\frac{|f(x)-f(y)|}{\|x-y\|}: x, y \in X, x \neq y\right\} .
$$

This space is denoted by $X^{\sharp}$ in [5, Chapter 7], where it is called the Lipschitz dual of $X$. The closed unit ball of the $\operatorname{space}^{\operatorname{Lip}_{0}}(X)$ is compact for the topology of pointwise convergence on $X$, and therefore this space has a canonical predual, namely the closed linear span of the linear forms $\delta(x)$ such that $\delta(x)(f)=f(x)$, where $x$ runs through $X$. We refer to [3] for uses of the map $\delta$ in a Fenchel duality context. This predual is defined and analysed in [29, Chapter 2] where it is called the Arens-Eells space. It turns out that this space enjoys remarkable functorial properties (see Section 2 below), quite reminiscent of those of free groups, which motivate the following definition.

Definition 1.1. Let $X$ be a Banach space, and let $\operatorname{Lip}_{0}(X)$ be the Banach space of real-valued Lipschitz functions on $X$ which vanish at 0 . The Lipschitz-free space over $X$, denoted by $\mathcal{F}(X)$, is the canonical predual

2000 Mathematics Subject Classification: Primary 46B20; Secondary 46B26, 46B28.

The second author was supported by NSF grant DMS-9870027. 
of $\operatorname{Lip}_{0}(X)$, that is, the norm closed linear subspace of $\operatorname{Lip}_{0}(X)^{*}$ spanned by the evaluation functionals $\delta(x)$ with $x \in X$.

We shall refer to $\mathcal{F}(X)$ as the free space over $X$. We note that such a notion has been investigated before in the frame of topological vector spaces [11]. Our purpose in considering the free spaces is to investigate the following general problem: if $X$ and $Y$ are Lipschitz isomorphic Banach spaces, that is, if there exists a bijective and bi-Lipschitz map $F: X \rightarrow Y$, are $X$ and $Y$ linearly isomorphic? It is known that the answer to this question is negative in full generality, but it remains an important open problem in the separable case. We refer to the authoritative book [5] for this topic and related matters.

Free spaces are naturally relevant to this problem. Indeed, it is clear that the spaces $\operatorname{Lip}_{0}(X)$ and $\mathcal{F}(X)$ are Lipschitz invariants. Moreover, the map $\delta$ defines a nonlinear isometric embedding from $X$ into $\mathcal{F}(X)$, with a linear left inverse. Finally, moving to free spaces allows linearization of Lipschitz maps and this opens the way for applications of the linear theory to nonlinear problems.

We now describe the contents of this paper. Section 2 gathers general results about free spaces. These results and their proofs are essentially algebraic. The crucial lifting property is introduced in Definition 2.7. Section 3 addresses the separable theory. It is shown there that every separable space has the lifting property. The main consequence of this result is that when a separable space $X$ embeds isometrically into a Banach space $Y$, then $Y$ contains a linear subspace isometric to $X$ (although a Lipschitz embedding does not provide in general a linear one, see [1]). Section 4 investigates the nonseparable theory. It turns out that the nonseparable case is completely different from the separable case, since for instance every nonseparable WCG space fails the lifting property. This provides many canonical examples of pairs of nonseparable spaces which are Lipschitz but not linearly isomorphic.

The standard examples of Banach spaces which are Lipschitz isomorphic but not linearly isomorphic were constructed by finding a Lipschitz section of a quotient map ([2], [9], [10], [5]). The results of Sections 3 and 4 together show that this method is simply not available for separable spaces, but provide a plethora of nonseparable examples. We refer to [8] and references therein for twisted sums and nonlinear methods.

It is shown in Section 5 that $X$ has the metric (respectively bounded) approximation property if and only if its free space $\mathcal{F}(X)$ has the metric (respectively, bounded) approximation property. An application is that the bounded approximation property is invariant under Lipschitz isomorphisms.

This work contributes to support the feeling that the separable spaces "behave well" with respect to Lipschitz isomorphisms, and that very subtle differentiabilty statements could provide in many (in all?) separable cases 
the identification of the Lipschitz isomorphism class with the linear one, already shown for most of the classical spaces (see [18], [16]). We refer to [17] and [23] for recent and deep differentiability theorems.

The methods used here can also be applied to study uniform homeomorphisms and even isomorphisms of nets. This theory is a little different because infinitesimal arguments are not available any more in this context. We refer to the forthcoming paper [21].

2. Free spaces and diagrams. This section contains the basic facts concerning the behavior of free spaces with respect to commutative diagrams. Some of these facts are well known (see [20], [29]) but we recall the simple proofs and fix the notation.

Let $X$ be a real Banach space (all Banach spaces will be assumed real). First we observe that $\mathcal{F}(X)$ has the same density character as $X$; in particular if $X$ is separable then so is $\mathcal{F}(X)$.

We denote by $\delta=\delta_{X}: X \rightarrow \mathcal{F}(X)$ the canonical embedding so that $\langle\delta(x), f\rangle=f(x)$ for $f \in \operatorname{Lip}_{0}(X)$. It is sometimes convenient to think of $\mathcal{F}(X)$ as the completion of the set of Borel measures $\mu$ on $X$ with finite support under the norm

$$
\|\mu\|_{\mathcal{F}}=\sup _{\|f\|_{\text {Lip }} \leq 1} \int f d \mu .
$$

The following proposition states some obvious properties of $\delta$.

Proposition 2.1. The map $\delta: X \rightarrow \mathcal{F}(X)$ is a nonlinear isometry and is nowhere Gateaux differentiable.

Proof. We only need to remark that if $t \mapsto \delta(u+t v)$ has any point of differentiability at 0 then it would follow that for every $f \in \operatorname{Lip}_{0}(X)$ we would have differentiability of $t \mapsto f(u+t v)$ at 0 . Consider the map $f(x)=\|x-u\|-\|u\|$.

It now follows that any finite Borel measure $\mu$ supported on a compact subset $K$ of $X$ can be identified with a member of $\mathcal{F}(X)$. Indeed,

$$
\mu=\int_{K} \delta(x) d \mu(x)
$$

as a Bochner integral.

Lemma 2.2. Let $X$ and $Y$ be Banach spaces and suppose $L: X \rightarrow Y$ is a Lipschitz map such that $L(0)=0$. There exists a unique linear map $\widehat{L}: \mathcal{F}(X) \rightarrow \mathcal{F}(Y)$ such that $\widehat{L} \delta_{X}=\delta_{Y} L$, i.e. the following diagram commutes: 


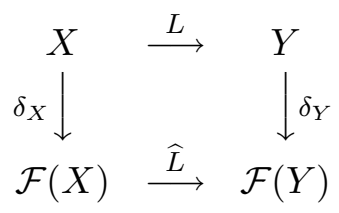

and $\|\widehat{L}\|=\|L\|_{\text {Lip }}$ where $\|\cdot\|_{\text {Lip }}$ denotes the Lipschitz norm of $L$.

Proof. The linear map $L^{\#}: \operatorname{Lip}_{0}(Y) \rightarrow \operatorname{Lip}_{0}(X)$ defined by $L^{\#}(F)=$ $F \circ L$ is pointwise-to-pointwise continuous, hence there is a linear map $\widehat{L}$ between the preduals such that $\widehat{L}^{*}=L^{\#}$. It is clear that $\left\|L^{\#}\right\|=\operatorname{Lip}(L)$, and $\|\widehat{L}\|=\left\|\widehat{L}^{*}\right\|=\left\|L^{\#}\right\|$. The other assertions are clear.

Note that it is straightforward to check that $\widehat{S T}=\widehat{S} \widehat{T}$ in full generality. One special case should be singled out.

Lemma 2.3. If $X$ is a subspace of $Y$ and $\iota: X \rightarrow Y$ is the canonical embedding then $\widehat{\iota}: \mathcal{F}(X) \rightarrow \mathcal{F}(Y)$ is an isometric embedding.

Proof. Suppose $\mu$ is a finitely supported measure on $X$. Choose $f \in$ $\operatorname{Lip}_{0}(X)$ with $\int f d \mu=\|\mu\|_{\mathcal{F}(X)}$ and $\|f\|_{\text {Lip }}=1$. Then $f$ has an extension to $g \in \operatorname{Lip}_{0}(Y)$ with $\|g\|_{\text {Lip }}=1$. It follows that $\|\widehat{\iota} \mu\|_{\mathcal{F}(Y)}=\|\mu\|_{\mathcal{F}(X)}$.

Lemma 2.3 allows one to consider $\mathcal{F}(X)$ as a subspace of $\mathcal{F}(Y)$ whenever $X$ is a subspace of $Y$.

Let us make a remark on typical applications of Lemmas 2.2 and 2.3. Suppose $X$ and $Y$ are Banach spaces. Suppose there are Lipschitz maps $L: X \rightarrow Y$ and $F: Y \rightarrow X$ such that $F L=\operatorname{Id}_{X}$ (equivalently $X$ is Lipschitz isomorphic to a subset of $Y$ onto which there is a Lipschitz retraction). Then $\mathcal{F}(X)$ is isomorphic to a complemented subspace of $\mathcal{F}(Y)$ by considering $\widehat{F}$ and $\widehat{L}$. In the case when $X$ is simply a subspace of $Y$ and there is a Lipschitz retraction of $Y$ onto $X$ then $\mathcal{F}(X)$ is a complemented subspace of $\mathcal{F}(Y)$.

If $\mu$ is a measure of finite support on $X$ we can define its barycenter $\beta(\mu)=\beta_{X}(\mu) \in X$ by

$$
\beta(\mu)=\int x d \mu .
$$

Note that if $x^{*} \in X^{*}$ we have

$$
\left|\left\langle\beta(\mu), x^{*}\right\rangle\right| \leq\left\|x^{*}\right\|\|\mu\|_{\mathcal{F}}
$$

and so $\beta$ extends to a bounded linear operator $\beta: \mathcal{F}(X) \rightarrow X$. The following lemma is clear.

Lemma 2.4. Let $X$ be a Banach space. Then $\beta$ is a linear quotient map $\mathcal{F}(X) \rightarrow X$ and is a left inverse of $\delta$, i.e. $\beta \delta=\operatorname{Id}_{X}$.

If we define $\bar{L}=\beta_{Y} \widehat{L}$, we deduce from Lemma 2.2 the following 
Lemma 2.5. Let $L$ be a Lipschitz map from a Banach space $X$ to a Banach space $Y$ such that $L(0)=0$. There exists a unique linear map $\bar{L}$ : $\mathcal{F}(X) \rightarrow Y$ such that $\bar{L} \delta_{X}=L$, and $\|\bar{L}\|=\|L\|_{\text {Lip }}$.

Now suppose

$$
0 \rightarrow Z \stackrel{R}{\rightarrow} Y \stackrel{S}{\rightarrow} X \rightarrow 0
$$

is a short exact sequence. We recall that this sequence splits if there is a bounded linear map $V: X \rightarrow Y$ so that $S V=\mathrm{Id}_{X}$, or equivalently there is a bounded linear map $W: Y \rightarrow Z$ so that $W R=\operatorname{Id}_{Z}$. We shall say that the sequence Lipschitz splits if there is a Lipschitz, but not necessarily linear, map $L: X \rightarrow Y$ so that $S L=\operatorname{Id}_{X}$. We can always assume that $L(0)=0$.

Let $Z_{X}=\beta_{X}^{-1}(0)$ be the kernel of $\beta_{X}$. Then Lemma 2.4 can be interpreted as saying that the short exact sequence of Banach spaces

$$
0 \rightarrow Z_{X} \rightarrow \mathcal{F}(X) \stackrel{\beta}{\rightarrow} X \rightarrow 0
$$

Lipschitz splits. In fact $\delta$ is an isometric Lipschitz lifting for $\beta$. Our next statement goes back to the seminal paper [22].

Proposition 2.6. If the short exact sequence

$$
0 \rightarrow Z \stackrel{R}{\rightarrow} Y \stackrel{S}{\rightarrow} X \rightarrow 0
$$

Lipschitz splits then the dual sequence

$$
0 \rightarrow X^{*} \stackrel{S^{*}}{\rightarrow} Y^{*} \stackrel{R^{*}}{\rightarrow} Z^{*} \rightarrow 0
$$

splits (linearly). Moreover in the isometric case, if $R$ is a linear isometry, $S$ is a linear quotient map and there is an isometric Lipschitz section $L$ : $X \rightarrow Y$, then there is a linear isometry $V: Y^{*} \rightarrow X^{*}$ with $\|V\|=1$ and $V S^{*}=\operatorname{Id}_{X^{*}}$.

Proof. Let $L: X \rightarrow Y$ be a Lipschitz section. We recall that by [5, Proposition 7.5] there is a contractive linear projection $P: \operatorname{Lip}_{0}(X) \rightarrow X^{*}$. Let $L^{\#}: \operatorname{Lip}_{0}(Y) \rightarrow \operatorname{Lip}_{0}(X)$ be defined by $L^{\#} f=f \circ L$. Then $P L^{\#}$ : $Y^{*} \subset \operatorname{Lip}_{0}(Y) \rightarrow X^{*}$ is a linear map and $P L^{\#} S^{*}=\operatorname{Id}_{X^{*}}$. The remaining statements are clear.

Proposition 2.6 implies that if a short exact sequence Lipschitz splits, then its bidual sequence splits. In particular, when the spaces involved are reflexive, Lipschitz splitting and linear splitting are equivalent. Also, a Lipschitz splitting implies that $X$ is isomorphic to a subspace of $Y^{* *}$. This last statement follows, when $X$ is separable, from [5, Corollary 7.10], which follows by a Gateaux differentiation technique. We will see below that in the separable case one can do better and $X$ actually embeds into $Y$ in this setting. 
We now define a new concept which turns out to be crucially important for our purpose.

Definition 2.7. A Banach space $X$ has the (isometric) Lipschitz-lifting property if there exists a (norm one) continuous linear map $T: X \rightarrow \mathcal{F}(X)$ such that $\beta T=\operatorname{Id}_{X}$.

We shall refer to the Lipschitz-lifting property as the lifting property.

Proposition 2.8. Let $X$ be a Banach space. Then $X$ has the lifting property if and only if every short exact sequence

$$
0 \rightarrow Z \rightarrow Y \rightarrow X \rightarrow 0
$$

which Lipschitz splits also linearly splits.

Proof. We show that a short exact sequence

$$
0 \rightarrow Z \stackrel{R}{\rightarrow} Y \stackrel{S}{\rightarrow} X \rightarrow 0
$$

which Lipschitz splits is a pushout of the short exact sequence

$$
0 \rightarrow Z_{X} \rightarrow \mathcal{F}(X) \rightarrow X \rightarrow 0
$$

Indeed, let $L: X \rightarrow Y$ be a Lipschitz section. By Lemma 2.5 we can find a linear map $\bar{L}: \mathcal{F}(X) \rightarrow Y$ with $\bar{L} \delta_{X}=L$. Hence $S \bar{L} \delta_{X}=\operatorname{Id}_{X}$ from which it follows that $S \bar{L}=\beta_{X}$. Hence $S \bar{L}\left(Z_{X}\right)=\{0\}$ so that we can find an operator $V: Z_{X} \rightarrow Z$ so that the following diagram commutes:

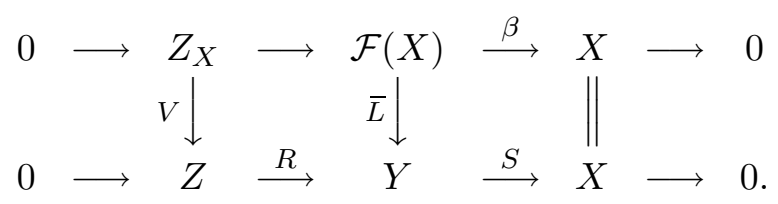

Now it is clear that if $T: X \rightarrow \mathcal{F}(X)$ is a linear lifting of $\beta$ then $\bar{L} T$ is a linear lifting of $S$.

The reasoning of Proposition 2.8 gives the following isometric version.

Proposition 2.9. Suppose $X$ is a Banach space with the isometric lifting property. Suppose $Y$ is a Banach space and $Q: Y \rightarrow X$ is a quotient map. Then if there exists a (not necessarily linear) isometry $L: X \rightarrow Y$ with $Q L=\operatorname{Id}_{X}$ then there is also a linear isometry $V: X \rightarrow Y$ with $Q V=\operatorname{Id}_{X}$.

The first examples of spaces which have the lifting property are provided by our next lemma.

Lemma 2.10. Let $X$ be an arbitrary Banach space. Then the free space $\mathcal{F}(X)$ over $X$ has the isometric lifting property.

Proof. Consider the isometry $\delta_{\mathcal{F}(X)} \delta_{X}: X \rightarrow \mathcal{F}(\mathcal{F}(X))=\mathcal{F}^{2}(X)$. By Lemma 2.5 there is a linear map $T: \mathcal{F}(X) \rightarrow \mathcal{F}^{2}(X)$ with $\|T\|=1$ and 
such that $T \delta_{X}(x)=\delta_{\mathcal{F}(X)} \delta_{X}(x)$. Thus $\beta_{\mathcal{F}(X)} T \delta_{X}(x)=\delta_{X}(x)$, from which it follows that $\beta_{\mathcal{F}(X)} T=\operatorname{Id}_{\mathcal{F}(X)}$.

Diagram chasing also provides the following fact.

Lemma 2.11. If $X$ has the lifting property and $Y$ is a subspace of $X$ onto which there is a continuous linear projection $\pi$, then $Y$ has the lifting property. If $X$ has the isometric lifting property and $\|\pi\|=1$ then $Y$ has the isometric lifting property.

Proof. If $\widehat{\pi}: \mathcal{F}(X) \rightarrow \mathcal{F}(Y)$ is the corresponding free extension, we have $\beta_{Y} \widehat{\pi} \delta_{X}=\pi$ and it follows since $\pi$ is linear that $\beta_{Y} \widehat{\pi}=\pi \beta_{X}$. If $T$ is a linear lifting of $X$, then $\beta_{Y} \widehat{\pi} T=\pi$ and thus the restriction of $\widehat{\pi} T$ to $Y$ is a linear lifting from $Y$ to $\mathcal{F}(Y)$. The second part is clear.

We are now ready to state the two main results of this section.

Theorem 2.12. Let $X$ be a Banach space. Then $\mathcal{F}(X)$ is Lipschitz isomorphic to the space $\mathcal{G}(X)=Z_{X} \oplus X$. Moreover, these two spaces are linearly isomorphic if and only if the space $X$ has the lifting property.

Proof. The map $L: \mathcal{F}(X) \rightarrow \mathcal{G}(X)$ defined by

$$
L(\mu)=\left(\mu-\delta_{X} \beta_{X}(\mu), \beta_{X}(\mu)\right)
$$

is easily seen to be a Lipschitz isomorphism between $\mathcal{F}(X)$ and $\mathcal{G}(X)$. If $T$ is a linear lifting of $X$, then we may replace $\delta_{X}$ by $T$ above to show that the spaces are linearly isomorphic. Conversely, if $\mathcal{F}(X)$ and $\mathcal{G}(X)$ are isomorphic, then $X$ is isomorphic to a complemented subspace of $\mathcal{F}(X)$, and thus by Lemmas 2.10 and 2.11 the space $X$ has the lifting property.

Proposition 2.8 already provides us with examples of spaces which fail the lifting property: indeed, it implies by [2] that for any uncountable set $\Gamma$, the space $c_{0}(\Gamma)$ fails the lifting property. We will see in Theorem 4.3 below that a much more general statement holds true.

Theorem 2.13. Suppose $X$ is a Banach space. Then $X$ has the isometric lifting property if and only if whenever $Y$ is a Banach space such that there exists a (not necessarily linear) isometry $L: X \rightarrow Y$ with $L(0)=0$ and $Y=\overline{\operatorname{span}}(L(X))$ then $Y$ contains a contractively complemented subspace linearly isometric to $X$.

Proof. If $X$ has the isometric lifting property and $L: X \rightarrow Y$ is an isometry with the linear span of $L(X)$ dense, then a theorem of Figiel [13] asserts the existence of a quotient map $Q: Y \rightarrow X$ so that $Q L=\operatorname{Id}_{X}$. The conclusion then follows from Proposition 2.9. On the other hand if $X$ has the latter property we take $Y=\mathcal{F}(X)$ and deduce that $X$ is contractively complemented in $\mathcal{F}(X)$ and hence has the isometric lifting property. 
3. Liftings of separable spaces. The main result of this section is Theorem 3.1 below. Its proof should be compared with the proof of [5, Proposition 7.5] as it in some sense consists in localizing the invariant means on a compact subset of $X$. Theorem 3.1 can also be viewed as a weak differentiability result. We have seen, however, that the natural Lipschitz map $\delta: X \rightarrow \mathcal{F}(X)$ is nowhere Gateaux differentiable, and that $\mathcal{F}(X)$ contains an isometric copy of the space $L_{1}=\mathcal{F}(\mathbb{R})$ and thus fails in particular the Radon-Nikodym property.

We now state and prove

THEOREM 3.1. Every separable Banach space has the isometric lifting property.

Proof. Let $\left\{x_{n}\right\}$ be a linearly independent sequence in $X$ which spans a dense linear subspace $D$ of $X$, and such that the set

$$
K=\left\{\sum_{n=1}^{\infty} t_{n} x_{n}: 0 \leq t_{n} \leq 1\right\}
$$

is a norm compact subset of $X$. We denote by $H=[0,1]^{\mathbb{N}}$ the Hilbert cube, and by $\lambda$ the product of the Lebesgue measures on every factor. Similarly, we let $\mathbb{N}_{n}=\mathbb{N} \backslash\{n\}, H_{n}=[0,1]^{\mathbb{N}_{n}}$, and $\pi_{n}$ be the natural restriction map from $H$ onto $H_{n}$. We let $\lambda_{n}=\pi_{n}(\lambda)$. For $t=\left(t_{n}\right) \in H$, we let

$$
L(t)=\sum_{k=1}^{\infty} t_{k} x_{k}
$$

and we define $S_{n}: H_{n} \rightarrow X$ by

$$
S_{n}(t)=\sum_{k \neq n} t_{k} x_{k}
$$

With this notation, we now define $\phi_{n} \in \mathcal{F}(X)$ by the Bochner integral

$$
\phi_{n}=\int_{H_{n}}\left(\delta\left(x_{n}+S_{n}(t)\right)-\delta\left(S_{n}(t)\right)\right) d \lambda_{n}(t)
$$

and we denote by $R_{D}$ the linear map from $D$ to $\mathcal{F}(X)$ which satisfies $R_{D}\left(x_{n}\right)=\phi_{n}$. Since $\lambda_{n}$ is a probability measure, we clearly have $\beta\left(\phi_{n}\right)=x_{n}$ and thus $\beta R_{D}=\operatorname{Id}_{D}$. If $f$ is a Lipschitz Gateaux differentiable function on $X$, we have

$$
f\left(x_{n}+S_{n}(t)\right)-f\left(S_{n}(t)\right)=\int_{0}^{1}\left\langle\nabla f\left(u x_{n}+S_{n}(t)\right), x_{n}\right\rangle d u
$$

and it follows from (3.1) and Fubini's theorem that for such functions

$$
\left\langle f, \phi_{n}\right\rangle=\int_{H}\left\langle\nabla f(L(t)), x_{n}\right\rangle d \lambda(t),
$$


and thus for all $x \in D$ we have

$$
\left\langle f, R_{D}(x)\right\rangle=\int_{H}\langle\nabla f(L(t)), x\rangle d \lambda(t) .
$$

Note we have the estimate $\|\nabla f(y)\|_{X^{*}} \leq\|f\|_{\text {Lip }}$ and so

$$
\left|\left\langle f, R_{D}(x)\right\rangle\right| \leq\|f\|_{\text {Lip }}\|x\| .
$$

By [5, Cor. 6.43], the subset of the unit ball of $\operatorname{Lip}_{0}(X)$ consisting of the Gateaux differentiable functions is uniformly dense in this unit ball, and in particular weak* dense. It follows that (3.2) implies that

$$
\left\|R_{D}(x)\right\|_{F} \leq\|x\|
$$

for every $x \in D$, and thus the linear map $R_{D}$ extends to a linear map $R$ of norm 1 from $X$ to $\mathcal{F}(X)$ which clearly satisfies $\beta R=\operatorname{Id}_{X}$.

What we do in the above proof is differentiate the convolution of the Dirac lifting $\delta$ with a cube measure. Differentiable measures can also be used for this purpose: indeed, if $F: X \rightarrow Y$ is a Lipschitz map from a separable Banach space $X$ to a Banach space $Y$ and if $\gamma$ is a nondegenerate Gaussian probability measure on $X$ with mean 0 (see [5, Chapter 6$]$ ), then for every $y^{*} \in Y^{*}$, the real-valued map

$$
\left(y^{*} \circ F\right) * \gamma(x)=\int_{X} y^{*} \circ F(x+t) d \gamma(t)
$$

is Gateaux differentiable everywhere on $X$ (see e.g. the proof of Theorem 7 in [7]). If $G\left(y^{*}\right) \in X^{*}$ denotes the Gateaux derivative of this map at $x=0$, it is clear that $G$ is linear in $y^{*}$ and has norm less than the Lipschitz constant of $F$. By [6, Theorem 5.1.8], for all $h$ in the dense subspace $H(\gamma)$ of $X$ we have

$$
G\left(y^{*}\right)(h)=\int_{X} y^{*} \circ F(t) d \nu_{h}(t)
$$

for some measure $\nu_{h}$ on $X$, and Lebesgue's dominated convergence theorem shows that $G$ is weak*-to-weak* continuous. Hence there is a bounded linear operator $R$ from $X$ to $Y$ with $G=R^{*}$. This linear operator $R$ is the weak Gateaux derivative of $F * \gamma$ at $x=0$. If $T: Y \rightarrow X$ is a bounded linear left inverse to $F$, a straightforward computation shows that $T R=\operatorname{Id}_{X}$. This applies in particular to $F=\delta$ and provides an alternative proof of Theorem 3.1.

Corollary 3.2. Let $Y$ be a Banach space, and $Q: Y \rightarrow X$ be a quotient map from $Y$ onto a separable Banach space $X$. If $Q$ has a Lipschitz right inverse $L$, then $Q$ has a linear right inverse $V$ with $\|V\|=\|L\|_{\text {Lip. }}$.

Proof. This follows immediately from Theorem 3.1 and Proposition 2.8. 
Theorem 3.1 has an application to the results of Figiel on isometric embeddings. The following corollary combined with Corollary 4.4 below answers a problem from [12] (see [14, Question 1.6.2]).

Corollary 3.3. Let $X$ be a separable Banach space. If there exists an isometric embedding from $X$ into a Banach space $Y$, then $Y$ contains a linear subspace which is isometric to $X$.

Proof. We may and do assume that $L(0)=0$, and then the result follows from Theorem 2.13.

We refer to [25] for an explicit linear embedding when the spaces $X$ and $Y$ are $\mathcal{C}(K)$ spaces. Note that we cannot replace "isometric embedding" by "Lipschitz embedding" in Corollary 3.3. Indeed, any separable Banach space can be Lipschitz embedded into $c_{0}(\mathbb{N})([1]$; see [26] for a quantitative improvement) but few of them are isomorphic to subspaces of $c_{0}(\mathbb{N})$. The proof of Corollary 3.3 relies heavily on Figiel's theorem [13]. We will see in Proposition 4.5 below that this theorem admits a converse, and thus Corollary 3.3 requires the existence of some isometric embedding, and is truly separable as well (see the comments after Corollary 4.4).

We recall that for every closed subspace $F$ of a Banach space $Y$, the quotient map $Q: Y \rightarrow Y / F$ admits a continuous positive homogeneous lifting (see [5, Proposition 1.19]). However we have:

Corollary 3.4. Let $Y$ be a Banach space, and let $F$ be a closed subspace such that $Y / F$ is separable. If there exists a uniformly continuous positive homogeneous right inverse to the quotient $\operatorname{map} Q: Y \rightarrow Y / F$, then $F$ is linearly complemented in $Y$.

Proof. It is easily checked that a positive homogeneous map is Lipschitz as soon as it is uniformly continuous. The result then follows from Corollary 3.2 .

Note that the above statement fails in general when the right inverse is not homogeneous. Indeed, [21] provides canonical examples of linear quotient maps between separable Banach spaces with a uniformly continuous right inverse but no linear continuous right inverse.

4. Nonseparable spaces. We will show in this section that the nonseparable theory is completely different from the separable one. This will in particular provide us with canonical examples of nonseparable Banach spaces which are Lipschitz but not linearly isomorphic.

The following technical result will easily imply the main result of this section.

Proposition 4.1. Let $X$ be a weakly compactly generated Banach space. Then every weakly compact subset of $\mathcal{F}(X)$ is separable. 
Proof. We first note that if $V$ is a separable Banach space, the subset of $\operatorname{Lip}(V)$ consisting of all weakly continuous functions is norming (to check this, use Lemma 2.3 and embed $V$ in $C[0,1]$, which has a monotone basis, and consider functions $F \circ P_{n}$ where $P_{n}$ is the $n$th basis projection).

Lemma 4.2. Let $W$ be a weakly compact subset of $X$ and suppose $\left(\mu_{n}\right)$ is a sequence of measures of finite support in $W$ with $\sup \left|\mu_{n}\right|(W)=K$ $<\infty$. Suppose $\inf _{m \neq n}\left\|\mu_{n}-\mu_{m}\right\|_{\mathcal{F}}>\varepsilon$. Then there exists $f \in \operatorname{Lip}(X)$ with $\|f\|_{\text {Lip }} \leq 1$ and an infinite subset $\mathbb{M}$ of $\mathbb{N}$ so that

$$
\left|\int f d \mu_{n}\right| \geq \frac{1}{8} \varepsilon, \quad n \in \mathbb{M} .
$$

Proof. By using Lemma 2.3 we may and do assume that $X$ is separable. We may assume that $\sup \{\|x\|: x \in W\}=A<\infty$. Then we also have $\sup \left\{\left\|\mu_{n}\right\|_{\mathcal{F}}\right\} \leq A K$.

We first pick a subsequence $\left(\nu_{n}\right)$ of $\left(\mu_{n}\right)$ inductively. Let $S_{n}$ be the closed convex hull of the union of the supports of $\nu_{1}, \ldots, \nu_{n-1}$. Let $E_{n}$ be the closed absolutely convex hull of the set $\left\{\delta(x): x \in S_{n}\right\}$. Then $E_{n}$ is compact in $\mathcal{F}(X)$. It is clear that we can pick a subsequence so that

$$
d_{\mathcal{F}}\left(\nu_{n}, b 2^{n} E_{n-1}\right)>c>\frac{1}{2} \varepsilon
$$

where $b=16 K^{2} A \varepsilon^{-1}$. By a Hahn-Banach argument we may find a Lipschitz function $f_{n}$ with constant one so that

$$
\int f_{n} d \mu_{n}>c+b 2^{n} \int f_{n} d \gamma
$$

for $\gamma \in E_{n-1}$. This implies that

$$
\left|\int f_{n} d \gamma\right| \leq K A b^{-1} 2^{-n}, \quad \gamma \in E_{n-1} .
$$

Next we use the fact that the set of weakly continuous functions in $\operatorname{Lip}_{0}(X)$ is norming. It follows that we may replace each $f_{n}$ by a weakly continuous function with $\left\|f_{n}\right\|_{\text {Lip }} \leq 1$ and have the same inequalities valid. Hence we assume each $f_{n}$ is weakly continuous.

We can further assume, by passing to a subsequence, that $\left|\nu_{n}\right|$ is weak* convergent to some $\nu \in C(W)^{*}$, where $W$ is equipped with the weak topology. If $k<n$,

$$
\int\left|f_{n}\right| d\left|\nu_{k}\right|=\int_{f_{n}>0} f_{n} d\left|\nu_{k}\right|-\int_{f_{n}<0} f_{n} d\left|\nu_{k}\right| .
$$

Observe that both $\chi_{f_{n}>0}\left|\nu_{k}\right|$ and $\chi_{f_{n}<0}\left|\nu_{k}\right|$ belong to $K E_{n-1}$ and so by (4.3) we have an estimate

$$
\int\left|f_{n}\right| d\left|\nu_{k}\right| \leq 2 K^{2} A b^{-1} 2^{-n}, \quad k<n .
$$


From (4.2) we also have

$$
\int f_{n} d \nu_{n}>c, \quad n \in \mathbb{N} .
$$

Let $S$ be the norm closure of $\bigcup_{n=1}^{\infty} S_{n} \subset X$. Note that $S$ contains the support of $\nu$ by Mazur's theorem. Furthermore $\lim _{n \rightarrow \infty} f_{n}(x)=0$ for $x \in S$. Hence $f_{n}(x) \rightarrow 0$ on the support of $\nu$. Thus

$$
\lim _{n \rightarrow \infty} \int\left|f_{n}\right| d \nu=0
$$

Now since each $f_{n}$ is weakly continuous,

$$
\lim _{k \rightarrow \infty} \int\left|f_{n}\right| d\left|\nu_{k}\right|=\int\left|f_{n}\right| d \nu
$$

Thus passing to a subsequence we can suppose that

$$
\int\left|f_{n}\right|\left|d \nu_{k}\right|<2 K^{2} A b^{-1} 2^{-k} \quad \text { for } k \neq n .
$$

Now for each $n$ let $h_{n}=\max \left(f_{n}, 0\right)$ or $h_{n}=\max \left(-f_{n}, 0\right)$ in such a way that

$$
\left|\int h_{n} d \mu_{n}\right| \geq \frac{1}{2} c \text {. }
$$

Finally, let $f=\sup _{n} h_{n}$. Then $\|f\|_{\text {Lip }} \leq 1$ and

$$
0 \leq f-h_{n} \leq \sum_{k \neq n} h_{k}
$$

Thus from (4.6),

$$
\left|\int f d \mu_{n}-\int h_{n} d \nu_{n}\right| \leq 2 K^{2} A b^{-1}=\frac{1}{8} \varepsilon .
$$

The result follows.

We return to the proof of Proposition 4.1. Suppose that $X$ is an arbitrary WCG space. Assume that there exists $\varepsilon>0$ and an uncountable relatively weakly compact subset $\left(\nu_{i}\right)$ in $\mathcal{F}(X)$ with $\left\|\nu_{i}-\nu_{j}\right\|>\varepsilon$ if $i \neq j$. Let $W$ be a weakly compact subset of $X$ such that $\bigcup n W$ is dense in $X$. For each $m \in \mathbb{N}$ let $C_{m} \subset \mathcal{F}(X)$ be the set of all $\mu$ with finite support in $m W$ and with $|\mu|(m W) \leq m$. Then for each $i, \lim _{m \rightarrow \infty} d\left(\nu_{i}, C_{m}\right) \rightarrow 0$. It follows that we can find an infinite countable set $\mathbb{M}$ and a fixed $m$ so that $d\left(\nu_{i}, C_{m}\right)<\varepsilon / 26$ for $i \in \mathbb{M}$. Pick $\mu_{i} \in C_{m}$ so that $\left\|\nu_{i}-\mu_{i}\right\|_{F}<\varepsilon / 26$. Note that $\left\|\mu_{i}-\mu_{j}\right\|>$ $12 \varepsilon / 13$ when $i \neq j$.

We can suppose, by passing to a subsequence, that $\left(\nu_{i}\right)$ converges weakly to some $\nu \in \mathcal{F}(X)$ and hence find $m_{1}$ and $\mu \in C_{m_{1}}$ so that $\|\nu-\mu\|_{F}<\varepsilon / 26$. Then by Lemma 4.2 we can find an infinite subset $\mathbb{M}_{0}$ of $\mathbb{M}$ and a function $f$ with Lipschitz constant one so that

$$
\left|\int f d\left(\mu_{i}-\mu\right)\right| \geq \frac{3}{26} \varepsilon, \quad i \in \mathbb{M}_{0} .
$$


However, since $\nu_{i} \rightarrow \nu$ weakly in $\mathcal{F}(X)$, we have

$$
\lim \left\langle\nu_{i}-\nu, f\right\rangle=0
$$

and hence for some $i$ we have

$$
\left|\int f d\left(\mu_{i}-\mu\right)\right|<\frac{1}{13} \varepsilon .
$$

This contradiction gives the result.

We can now prove the main result of this section.

THEOREM 4.3. A weakly compactly generated Banach space has the lifting property if and only if it is separable.

Proof. Let $X$ be a nonseparable WCG space. Let $W$ be a weakly compact subset which spans a dense linear subspace of $X$. If $T$ is a linear right inverse to the map $B_{X}$, then $T(W)$ is a weakly compact nonseparable subset of $\mathcal{F}(X)$, and this contradicts Proposition 4.1.

The following consequence provides us with a wealth of Lipschitz but not linearly isomorphic Banach spaces.

COROLlary 4.4. Let $X$ be a nonseparable weakly compactly generated Banach space. The space $\mathcal{F}(X)$ is Lipschitz isomorphic to the space $\mathcal{G}(X)=$ $Z_{X} \times X$, but $\mathcal{F}(X)$ does not contain a subspace isomorphic to $X$. In particular, $\mathcal{F}(X)$ and $\mathcal{G}(X)$ are not linearly isomorphic.

Proof. The first assertion was shown in Theorem 2.12. Since every weakly compact subset of $\mathcal{F}(X)$ is separable, this space does not contain a subspace isomorphic to $X$ and in particular $\mathcal{F}(X)$ and $\mathcal{G}(X)$ are not linearly isomorphic. Note that this last assertion also follows from Theorems 2.12 and 4.3.

Corollary 4.4 shows in particular that Corollary 3.3 fails in the nonseparable case: if $X$ is any nonseparable WCG space, then the map $\delta_{X}$ is an isometric embedding from $X$ into $\mathcal{F}(X)$, but $\mathcal{F}(X)$ does not contain a subspace isomorphic to $X$.

Note that it follows from Lemma 2.10 and Theorem 4.3 that if $X$ is not separable, then the space $\mathcal{F}(X)$ is not WCG. However, there exist two Lipschitz isomorphic but not linearly isomorphic WCG Banach spaces. This follows from a recent work of M. Bell and W. Marciszewski ([4]; see also [15]). The density character of these spaces is $\aleph_{\omega_{0}}$.

The space $\mathcal{F}(X)$ is a twisted sum of the two components of the space $\mathcal{G}(X)$. The first examples of Lipschitz isomorphic but not linearly isomorphic spaces have been constructed in this manner ([2]; see [9] for a generalization), which is natural in view of Figiel's theorem [13]. We now show that this theorem admits a converse. 
Proposition 4.5. Let $X$ and $Y$ be Banach spaces such that there exists a Lipschitz embedding $L: X \rightarrow Y$ such that $L(0)=0$ and $\overline{\operatorname{span}}(L(X))=Y$. Then $L$ admits a continuous linear left inverse $Q$ if and only if there exists an equivalent norm on $Y$ for which $L$ becomes an isometric embedding.

Proof. If there is such a norm, the existence of $Q$ follows from Figiel's theorem (see [5, Theorem 14.2]). Conversely, we assume that there is a continuous linear map $Q$ such that $Q L=\operatorname{Id}_{X}$. Using a first renorming of $Y$, we may and do assume that $\|Q\|=1$. Let $\bar{L}: \mathcal{F}(X) \rightarrow Y$ be the linear map obtained from Lemma 2.5. We denote the closed unit ball of a Banach space $Z$ by $B_{Z}$. We define an equivalent norm on $Y$ through its unit ball $B^{\prime}$ such that

$$
B^{\prime}=\overline{\operatorname{conv}}\left(\bar{L}\left(B_{\mathcal{F}(X)}\right) \cup B_{Y}\right) .
$$

It is clear that with this new norm one has $\|\bar{L}\|=1$ and thus $L$ becomes 1-Lipschitz. On the other hand, since $B_{\mathcal{F}(X)}$ is the convex hull of the "molecules" $(\delta(x)-\delta(y)) /\|x-y\|$ (see $\left[29\right.$, p. 38]) and $Q \bar{L} \delta=\operatorname{Id}_{X}$, we still have $Q\left(B^{\prime}\right)=B_{X}$ and thus the new norm of $Q$ is still 1 and it follows that $L$ is an isometric embedding from $X$ to $Y$ equipped with the new norm.

Proposition 4.5 allows us to find more examples of pairs of spaces $X$ and $Y$ such that $X$ can be isometrically embedded into a space $Y$ without being isomorphic to a subspace of $Y$ : using for instance [10, Lemma VI.8.10], we see that the $\mathcal{C}\left(K_{0}\right)$ space from [10, Example VI.8.7] is an Asplund space which can be renormed to contain an isometric copy of $c_{0}(\Gamma)$ with $\operatorname{Card}(\Gamma)=c$ although it does not contain an isomorphic linear copy of that space.

It is a natural question to ask which nonseparable spaces have the lifting property. We note that for any set $\Gamma$, the space $\ell_{1}(\Gamma)$ trivially has the lifting property since in fact any quotient map onto $\ell_{1}(\Gamma)$ has a linear right inverse. This can be generalized easily to uncountable $\ell_{1}$-sums of separable spaces. Moreover, by Lemma 2.10 the space $\mathcal{F}(X)$ has the lifting property for every Banach space $X$. On the other hand, we have:

Proposition 4.6. The space $\ell_{\infty}(\mathbb{N})$ fails the lifting property.

Proof. For $j \in \mathbb{N}$, let $e_{j} \in \ell_{\infty}(\mathbb{N})$ be such that $e_{j}(j)=1$ and $e_{j}(k)=0$ if $k \neq j$. Assume that there is a linear lifting $T$ of $\beta=\beta_{\ell_{\infty}}$. Then there exists a subspace $X$ of $\ell_{\infty}(\mathbb{N})$ such that $T\left(e_{j}\right) \in \mathcal{F}(X)$ for all $j$ and $X$ is isometric to a separable $\mathcal{C}(K)$ space. By [5, Theorem 1.6], $X$ is an absolute Lipschitz retract and thus by Lemma 2.2 the space $\mathcal{F}(X)$ is linearly complemented in $\mathcal{F}\left(\ell_{\infty}(\mathbb{N})\right)$ by a projection $P$. Since $\ell_{\infty}(\mathbb{N})$ is a Grothendieck space, the operator $P T$ is weakly compact, but this contradicts the fact that $\beta P T\left(e_{j}\right)=e_{j}$. 
Proposition 4.6 and Lemma 2.11 imply that all the spaces which contain $\ell_{\infty}(\mathbb{N})$ fail the lifting property. In particular, although $\ell_{\infty}(\mathbb{N})$ embeds isometrically into $\mathcal{F}\left(\ell_{\infty}(\mathbb{N})\right)$, it does not embed as a linear subspace.

We note that when a quotient map $Q: Y \rightarrow X$ between nonseparable spaces has a Lipschitz right inverse, then Lemma 2.3 and Theorem 3.1 show that every separable subspace of $X$ can be lifted to $Y$. The above results show that these partial liftings cannot usually be organized into a global lifting of $X$. However, there is a weaker statement which we will use later.

Proposition 4.7. Let $X$ be a Banach space and suppose $E$ is a finitedimensional subspace of $X$. Then there is a linear operator $W: X \rightarrow \mathcal{F}(X)^{* *}$ such that $\|W\|=1, \beta^{* *} W x=x$ for $x \in X$ and $W(E) \subset \mathcal{F}(X)$.

Proof. Let $e_{1}, \ldots, e_{n}$ be a basis of $E$. As in the proof of Theorem 3.1 there is a linear map $U: E \rightarrow \mathcal{F}(E) \subset \mathcal{F}(X)$ with $\|U\|=1$ and $\beta U e=e$ for $e \in E$ given by

$$
U e_{k}=\int_{0}^{1} \ldots \int_{0}^{1}\left(\delta\left(\sum_{j \neq k} t_{j} e_{j}+e_{k}\right)-\delta\left(\sum_{j \neq k} t_{j} e_{j}\right)\right) \prod_{j \neq k} d t_{j} .
$$

Now for every finite-dimensional subspace $G$ of $X$ containing $E$ and every $\varepsilon>0$ we will define a linear lifting $V_{G, \varepsilon}: G \rightarrow \mathcal{F}(G)$ with $\left\|V_{G, \varepsilon}\right\|=1$ and $\beta V_{G, \varepsilon} g=g$ for $g \in G$. We do this by defining a basis $g_{1}, \ldots, g_{m}$ of $G$ so that $g_{j}=e_{j}$ for $1 \leq j \leq n$ and $\sum_{j=n+1}^{m}\left\|g_{j}\right\|<\varepsilon$. Then $V_{G, \varepsilon}$ is defined by the same procedure, i.e.

$$
V_{G, \varepsilon} g_{k}=\int_{0}^{1} \ldots \int_{0}^{1}\left(\delta\left(\sum_{j \neq k} t_{j} g_{j}+g_{k}\right)-\delta\left(\sum_{j \neq k} t_{j} g_{j}\right)\right) \prod_{j \neq k} d t_{j} .
$$

Then for $1 \leq j \leq n$ we have

$$
\left\|V_{G, \varepsilon} e_{j}-U e_{j}\right\|_{\mathcal{F}} \leq 2 \varepsilon .
$$

Extend $V_{G, \varepsilon}$ to a nonlinear map $\widetilde{V}_{G, \varepsilon}: X \rightarrow \mathcal{F}(X)$ by setting $V_{G, \varepsilon} x=0$ if $x \notin G$. By a standard compactness argument we may find a subnet of the net $\left\{V_{G, \varepsilon}: G \supset F, \varepsilon>0\right\}$ which converges pointwise weak* to some $W$ : $X \rightarrow \mathcal{F}(X)^{* *}$. Then $W$ is clearly a linear operator of norm one, $\beta^{* *} W x=x$ for $x \in X$ and $W e=U e$ for $e \in E$.

5. Approximation properties. We recall that a Banach space $X$ has the $\lambda$-bounded approximation property $(\lambda$-(BAP) $)$ if for every compact set $K \subset X$ and every $\varepsilon>0$ there exists a finite-rank operator $T: X \rightarrow X$ with $\|T\| \leq \lambda$ and $\|T x-x\|<\varepsilon$ for $x \in K$. The 1-(BAP) is called the metric approximation property (MAP). If $X$ has $\lambda$-(BAP) for some $\lambda$ we say that $X$ has (BAP). 
Proposition 5.1. Let $E$ be a finite-dimensional Banach space. Then $\mathcal{F}(E)$ has the metric approximation property $(M A P)$.

Proof. We will show that given $\varepsilon>0$ there is a sequence $L_{n}$ of Lipschitz maps $L_{n}: E \rightarrow \mathcal{F}(E)$ with finite-dimensional range and $L(0)=0$ such that $\lim _{n \rightarrow \infty}\left\langle L_{n}(x), f\right\rangle=f(x)$ for $x \in B_{E}$ and $\limsup _{n \rightarrow \infty}\left\|L_{n}\right\|_{\text {Lip }}<1+\varepsilon$. Let us first observe that this will suffice to show the result. First we note that $L_{n} x$ converges weakly to $\delta(x)$ and so by a convex combination argument we can suppose we actually have strong convergence. Once this is done we can find a sequence $H_{n}$ of Lipschitz maps $H_{n}: E \rightarrow \mathcal{F}(E)$ with finite-dimensional range such that $H(0)=0,\left\|H_{n}\right\|_{\text {Lip }}<1+1 / n$ and $\left\|H_{n} x-\delta(x)\right\|<1 / n^{2}$ for $x \in B_{E}$. Let $F_{n}(x)=\Lambda_{n} H_{n}(x / n)$ where $\Lambda_{n}: \mathcal{F}(E) \rightarrow \mathcal{F}(E)$ is the linear map induced by the Lipschitz map $\delta(x) \mapsto \delta(n x)$. Observe that $\left\|F_{n}\right\|_{\text {Lip }}<$ $1+1 / n$ and $\left\|F_{n}(x)-\delta(x)\right\|<1 / n$ for $x \in n B_{E}$. Now it easy to see that $\bar{F}_{n}: \mathcal{F}(E) \rightarrow \mathcal{F}(E)$ provide a sequence of finite-rank operators such that $\bar{F}_{n} \nu \rightarrow \nu$ for $\nu \in \mathcal{F}(E)$ and $\left\|\bar{F}_{n}\right\|<1+1 / n$.

In order to construct $L_{n}$, we use convolutions with Fejér kernels on cubes. We identify $E$ with $\mathbb{R}^{N}$ in such a way that $\|x\|_{\infty} \leq\|x\| \leq M\|x\|_{\infty}$ for some $M$ where $\|\cdot\|_{\infty}$ is the standard $\ell_{\infty}$-norm on $\mathbb{R}^{N}$. Let $B_{\infty}$ denote the unit ball of $\ell_{\infty}$. Suppose $a$ is chosen so large that $\log a>M \varepsilon^{-1}$. We write a typical element of $\mathbb{R}^{N}$ as $x=\left(x_{1}, \ldots, x_{N}\right)$ or $t=\left(t_{1}, \ldots, t_{N}\right)$; let $d t=d t_{1} \ldots d t_{N}$. Define

$$
\varphi(x)=\max \left(1-\frac{\log _{+}\|x\|_{\infty}}{\log a}, 0\right), \quad x \in \mathbb{R}^{N} .
$$

For $n \in \mathbb{N}$ define $\varrho_{n}: \mathbb{R}^{N} \rightarrow \mathcal{F}(E)$ by

$$
d \varrho_{n}(x)=\frac{1}{2^{3 N} a^{N} n^{N}}\left(\prod_{k=1}^{N} \frac{\sin ^{2} \frac{n \pi\left(x_{k}-t_{k}\right)}{8 a}}{\sin ^{2} \frac{\pi\left(x_{k}-t_{k}\right)}{8 a}}-\prod_{k=1}^{N} \frac{\sin ^{2} \frac{n \pi t_{k}}{8 a}}{\sin ^{2} \frac{\pi t_{k}}{8 a}}\right) \chi_{4 a B_{\infty}}(t) d t .
$$

(Thus $\varrho_{n}(x)$ is a compactly supported measure which is identified as an element of $\mathcal{F}(E)$.)

It is clear that $\varrho_{n}$ maps $\mathbb{R}^{N}$ into a finite-dimensional subspace of $\mathcal{F}(E)$. We next estimate the Lipschitz constant of $\varrho_{n}$ on the set $a B_{\infty}$. Suppose $\|x\|_{\infty},\|y\|_{\infty} \leq a$. Since we estimate a Lipschitz constant on a convex set, we may and do assume that $\|x-y\| \leq 2$. Let $u=\frac{1}{2}(x-y)$ and $v=\frac{1}{2}(x+y)$,

$$
d \mu=\frac{1}{2^{3 N} a^{N} n^{N}}\left(\prod_{k=1}^{N} \frac{\sin ^{2} \frac{n \pi\left(x_{k}-t_{k}\right)}{8 a}}{\sin ^{2} \frac{\pi\left(x_{k}-t_{k}\right)}{8 a}} \chi_{u+4 a B_{\infty}}(t)-\prod_{k=1}^{N} \frac{\sin ^{2} \frac{n \pi t_{k}}{8 a}}{\sin ^{2} \frac{\pi t_{k}}{8 a}} \chi_{4 a B_{\infty}}(t)\right) d t
$$

and

$$
d \nu=\frac{1}{2^{3 N} a^{N} n^{N}}\left(\prod_{k=1}^{N} \frac{\sin ^{2} \frac{n \pi\left(y_{k}-t_{k}\right)}{8 a}}{\sin ^{2} \frac{\pi\left(y_{k}-t_{k}\right)}{8 a}} \chi_{-u+4 a B_{\infty}}(t)-\prod_{k=1}^{N} \frac{\sin ^{2} \frac{n \pi t_{k}}{8 a}}{\sin ^{2} \frac{\pi t_{k}}{8 a}} \chi_{4 a B_{\infty}}(t)\right) d t .
$$

Then if $f \in \operatorname{Lip}_{0}(E)$ and $\|f\|_{\text {Lip }} \leq 1$ we have 


$$
\int f d \mu-\int f d \nu=\frac{1}{2^{3 N} a^{N} n^{N}} \int_{4 a B_{\infty}}(f(t+u)-f(t-u)) \prod_{k=1}^{N} \frac{\sin ^{2} \frac{n \pi\left(v_{k}-t_{k}\right)}{8 a}}{\sin ^{2} \frac{\pi\left(v_{k}-t_{k}\right)}{8 a}} d t .
$$

Hence

$$
\left|\int f d \mu-\int f d \nu\right| \leq\|x-y\|
$$

and so

$$
\|\mu-\nu\|_{\mathcal{F}} \leq\|x-y\| \text {. }
$$

Next we estimate $\left\|\varrho_{n}(x)-\mu\right\|_{\mathcal{F}}$. Suppose $f \in \operatorname{Lip}_{0}(E)$ and $\|f\|_{\text {Lip }} \leq 1$. Then, since $\|u\|_{\infty} \leq\|u\|$,

$$
\begin{aligned}
& \left|\int f d \varrho_{n}(x)-\int f d \mu\right| \\
& \leq \frac{1}{2^{3 N} a^{N} n^{N}} \int_{(4 a+\|u\|) B_{\infty} \backslash 4 a B_{\infty}}|f(t)| \prod_{k=1}^{N} \frac{\sin ^{2} \frac{n \pi\left(x_{k}-t_{k}\right)}{8 a}}{\sin ^{2} \frac{\pi\left(x_{k}-t_{k}\right)}{8 a}} d t .
\end{aligned}
$$

Now

$$
\begin{aligned}
& \int_{(4 a+\|u\|) B_{\infty} \backslash 4 a B_{\infty}}|f(t)| \prod_{k=1}^{N} \frac{\sin ^{2} \frac{n \pi\left(x_{k}-t_{k}\right)}{8 a}}{\sin ^{2} \frac{\pi\left(x_{k}-t_{k}\right)}{8 a}} d t \\
& \leq 5 a\left(\prod_{k=1}^{N} \int_{-(4 a+\|u\|)}^{4 a+\|u\|} \frac{\sin ^{2} \frac{n \pi\left(x_{k}-t_{k}\right)}{8 a}}{\sin ^{2} \frac{\pi\left(x_{k}-t_{k}\right)}{8 a}} d t_{k}-\prod_{k=1}^{N} \int_{-4 a}^{4 a} \frac{\sin ^{2} \frac{n \pi\left(x_{k}-t_{k}\right)}{8 a}}{\sin ^{2} \frac{\pi\left(x_{k}-t_{k}\right)}{8 a}} d t_{k}\right) \\
& \leq 5 \cdot 2^{3 N} a^{N+1}\left(\prod_{k=1}^{N} \int_{-(4 a+\|u\|) \pi /(4 a)}^{(4 a+\|u\|) \pi /(4 a)} \frac{\sin ^{2}\left(n\left(\frac{\pi x_{k}}{8 a}-\frac{\theta_{k}}{2}\right)\right)}{\sin ^{2}\left(\frac{\pi x_{k}}{8 a}-\frac{\theta_{k}}{2}\right)} \frac{d \theta_{k}}{2 \pi}\right. \\
& \left.-\prod_{k=1}^{N} \int_{-\pi}^{\pi} \frac{\sin ^{2}\left(n\left(\frac{\pi x_{k}}{8 a}-\frac{\theta_{k}}{2}\right)\right)}{\sin ^{2}\left(\frac{\pi x_{k}}{8 a}-\frac{\theta_{k}}{2}\right)} \frac{d \theta_{k}}{2 \pi}\right) \\
& \leq 5 \cdot 2^{3 N} a^{N+1}\left(\prod_{k=1}^{N\left[x_{k} /(4 a)-(4 a+\|u\|) /(4 a)\right] \pi} \int_{\sin ^{2}\left(\frac{\theta_{k}}{2}\right)}^{\left[x_{k} /(4 a)+(4 a+\|u\|) /(4 a)\right] \pi} \frac{\sin ^{2}\left(\frac{n \theta_{k}}{2 \pi}\right.}{\sin _{k}}\right. \\
& \left.-\prod_{k=1}^{N} \int_{-\pi}^{\pi} \frac{\sin ^{2}\left(\frac{n \theta_{k}}{2}\right)}{\sin ^{2}\left(\frac{\theta_{k}}{2}\right)} \frac{d \theta_{k}}{2 \pi}\right) \\
& \leq 5 \cdot 2^{3 N} a^{N+1}\left(\left(n+\frac{\|u\|}{2 a}\right)^{N}-n^{N}\right) .
\end{aligned}
$$

Here the last estimate follows from the fact that if $\pi \leq|\theta| \leq \pi+\frac{1}{4}\|u\| a^{-1} \pi$ then $\sin ^{2}\left(\frac{\pi x_{k}}{8 a}-\frac{\theta_{k}}{2}\right) \geq \frac{1}{2}$. 
Returning to (5.2) we have

$$
\left|\int f d \varrho_{n}(x)-\int f d \mu(x)\right| \leq 5\left(\left(1+\frac{\|u\|}{2 a n}\right)^{N}-1\right) a .
$$

Thus as $\|u\| \leq 1$,

$$
\left\|\varrho_{n}(x)-\mu\right\|_{\mathcal{F}} \leq 5 n^{-1} N 2^{N-2}\|u\| .
$$

We have a similar estimate on $\left\|\varrho_{n}(y)-\nu\right\|_{\mathcal{F}}$ and so

$$
\left\|\varrho_{n}(x)-\varrho_{n}(y)\right\| \leq c_{n}\|x-y\|
$$

where $c_{n}=1+5 n^{-1} N 2^{N-2}$.

Next let $L_{n}(x)=\varphi(x) \varrho_{n}(x)$. Again suppose $\|x\|_{\infty},\|y\|_{\infty} \leq a$ and suppose $\|x\|_{\infty} \leq\|y\|_{\infty}$. Then

$$
\begin{aligned}
\left\|L_{n}(x)-L_{n}(y)\right\|_{\mathcal{F}} & \leq|\varphi(y)| \cdot\left\|\varrho_{n}(y)-\varrho_{n}(x)\right\|_{\mathcal{F}}+|\varphi(y)-\varphi(x)| \cdot\left\|\varrho_{n}(x)\right\| \\
& \leq c_{n}\|x-y\|+(\log a)^{-1} \log \frac{\|y\|_{\infty}}{\|x\|_{\infty}} c_{n}\|x\| \\
& \leq c_{n}\left(\|x-y\|+\frac{\left(\|y\|_{\infty}-\|x\|_{\infty}\right)\|x\|}{\|x\|_{\infty} \log a}\right) \\
& \leq c_{n}\left(\|x-y\|+M(\log a)^{-1}\|x-y\|_{\infty}\right) \\
& \leq c_{n}\left(1+M(\log a)^{-1}\right)\|x-y\| .
\end{aligned}
$$

It is then easy to see that this estimate applies to all $x, y$, i.e.

$$
\left\|L_{n}\right\|_{\text {Lip }} \leq c_{n}\left(1+M(\log a)^{-1}\right) .
$$

Hence $\limsup _{n \rightarrow \infty}\left\|L_{n}\right\|_{\text {Lip }}<1+\varepsilon$. It is clear that if $x \in B_{E}$ then $x \in B_{\infty}$ and so $\varphi(x)=1$. Hence by standard Fourier series arguments

$$
\lim _{n \rightarrow \infty}\left\langle f, L_{n}(x)\right\rangle=f(x), \quad f \in \operatorname{Lip}_{0}(X) .
$$

This shows that $L_{n}$ satisfies our requirements and the proof is complete.

Definition 5.2. Let $X$ be a Banach space and $\lambda \geq 1$. Then we say that $X$ has the $\lambda$-Lipschitz bounded approximation property if for every compact set $K \subset X$ and $\varepsilon>0$ there exists a Lipschitz map $F: X \rightarrow X$ with finite-dimensional range such that $\|F\|_{\text {Lip }} \leq \lambda$ and $\|F(x)-x\| \leq \varepsilon$ for $x \in K$.

We remark that it is easy to see that we can assume $F(0)=0$ in the above definition.

THEOREM 5.3. Let $X$ be an arbitrary Banach space. Then the following conditions are equivalent: 
(1) $X$ has the $\lambda-(B A P)$.

(2) $\mathcal{F}(X)$ has the $\lambda-(B A P)$.

(3) $X$ has the $\lambda$-Lipschitz bounded approximation property.

Proof. It is trivial that (1) implies (3). Let us prove that (3) implies (2). To show $\mathcal{F}(X)$ has the $\lambda$-(BAP) it suffices by a density argument to show that if $\varepsilon>0$ and $x_{1}, \ldots, x_{n} \in X$ then there exists a finite rank linear map $T: \mathcal{F}(X) \rightarrow \mathcal{F}(X)$ such that $\left\|T\left(\delta\left(x_{j}\right)\right)-\delta\left(x_{j}\right)\right\|<\varepsilon$ for $1 \leq j \leq n$ and $\|T\| \leq \lambda$. To do this, note there is a finite-rank Lipschitz map $F: X \rightarrow X$ with $F(0)=0,\|F\|_{\text {Lip }} \leq \lambda$ and $\left\|F\left(x_{j}\right)-x_{j}\right\|<\varepsilon$ for $1 \leq j \leq n$. Let $\widehat{F}$ be the induced linear map as in Lemma 2.2. Then $\widehat{F}$ has range included in $\mathcal{F}(E)$ for some finite-dimensional subspace $E$ of $X$. By Proposition 5.1 this space has (MAP) and we can find a finite-rank linear operator $S: \mathcal{F}(E) \rightarrow \mathcal{F}(E)$ so that $\|S\| \leq 1$ and $\left\|S \widehat{F}\left(\delta\left(x_{j}\right)\right)-\delta\left(x_{j}\right)\right\|<\varepsilon$ for $1 \leq j \leq n$. Let $T=S \widehat{F}$ and we are done.

That (2) implies (1) is trivial if $X$ is separable by Theorem 3.1, which asserts that $X$ is isometric to a contractively complemented subspace of $\mathcal{F}(X)$. In the nonseparable case we use Proposition 4.7. Suppose $x_{1}, \ldots, x_{n}$ $\in X$ and $\varepsilon>0$. Let $E=\left[x_{1}, \ldots, x_{n}\right]$ be their linear span. Then we can find a linear operator $W: X \rightarrow \mathcal{F}(X)^{* *}$ with $\|W\|=1$ so that $\beta^{* *} W x=x$ for $x \in X$ and $W(E) \subset \mathcal{F}(X)$. Let $T: \mathcal{F}(X) \rightarrow \mathcal{F}(X)$ be a finite-rank operator with $\|T\| \leq \lambda$ and $\left\|T W x_{j}-W x_{j}\right\| \leq \varepsilon$ for $1 \leq j \leq n$. Then let $S=\beta^{* *} T^{* *} W$. Since $T^{* *}$ maps into $\mathcal{F}(X)$ we see that $S: X \rightarrow X$ is finite-rank and $\left\|S x_{j}-x_{j}\right\| \leq \varepsilon$ for $j=1, \ldots, n$.

Remark. Suppose $U$ is Pełczyński's universal basis space [27]. Then $\mathcal{F}(U)$ has (BAP) and hence ([19], [28]) embeds complementably into $U$. However, by Theorem 3.1, $U$ embeds complementably into $\mathcal{F}(U)$. Hence $\mathcal{F}(U)$ is isomorphic to $U$ by the standard Pełczyński decomposition technique.

TheOREm 5.4. Let $X$ be a Banach space with (BAP) and suppose $Y$ is a Banach space which is Lipschitz isomorphic to $X$. Then $Y$ has $(B A P)$.

Proof. The Lipschitz isomorphism induces a linear isomorphism between $\mathcal{F}(X)$ and $\mathcal{F}(Y)$. The conclusion follows from Theorem 5.3.

TheOREm 5.5. Let $X$ be a Banach space which is an absolute Lipschitz retract. Then $X$ has the bounded approximation property.

Proof. The space $X$ is a Lipschitz retract of $G=\ell_{\infty}\left(B_{X^{*}}\right)$. The space $G$ has (MAP), and thus $\mathcal{F}(G)$ has (MAP) by Theorem 5.3. By applying Lemma 2.2 to the retract of $G$ onto $X$, the space $\mathcal{F}(X)$ is complemented in $\mathcal{F}(G)$ and thus it has (BAP). By Theorem 5.3, $X$ also has (BAP). 


\section{References}

[1] I. Aharoni, Every separable metric space is Lipschitz equivalent to a subset of $c_{0}$, Israel J. Math. 19 (1974), 284-291.

[2] I. Aharoni and J. Lindenstrauss, Uniform equivalence between Banach spaces, Bull. Amer. Math. Soc. 84 (1978), 281-283.

[3] M. Bachir, A non-convex analogue to Fenchel duality, J. Funct. Anal. 181 (2001), 300-312.

[4] M. Bell and W. Marciszewski, On scattered Eberlein compact spaces, preprint, 2001.

[5] Y. Benyamini and J. Lindenstrauss, Geometric Nonlinear Functional Analysis, Vol. 1, Amer. Math. Soc. Colloq. Publ. 48, Amer. Math. Soc., 2000.

[6] V. I. Bogachev, Gaussian Measures, Math. Surveys Monogr. 62, Amer. Math. Soc., 1998.

[7] V. I. Bogachev and S. A. Shkarin, Differentiable and Lipschitzian mappings of Banach spaces, Mat. Zametki 44 (1988), 567-583 (in Russian); English transl.: Math. Notes 44 (1988), 790-798.

[8] F. Cabello Sánchez and J. M. F. Castillo, Banach space techniques underpinning a theory for nearly additive mappings, Dissertationes Math. 404 (2002).

[9] R. Deville, G. Godefroy and V. Zizler, The three space problem for smooth partitions of unity and $C(K)$ spaces, Math. Ann. 288 (1990), 613-625.

[10] - - - -, Smoothness and Renormings in Banach Spaces, Pitman Monogr. Surveys 64, Longman, 1993.

[11] J. Diestel, S. A. Morris and S. A. Saxon, Varieties of linear topological spaces, Trans. Amer. Math. Soc. 172 (1972), 207-230.

[12] L. Drewnowski, On nonlinear isometries between Banach spaces, in: Functional Analysis (Trier, 1994), S. Dierolf, S. Dineen and P. Domański (eds.), de Gruyter, Berlin, 1996, 129-141.

[13] T. Figiel, On nonlinear isometric embeddings of normed linear spaces, Bull. Acad. Polon. Sci. Sér. Sci. Math. Astronom. Phys. 16 (1968), 185-188.

[14] R. J. Fleming and J. E. Jamison, Isometries on Banach Spaces. Function Spaces, Monogr. Surveys Pure Appl. Math. 129, Chapman and Hall, Boca Raton, 2003.

[15] G. Godefroy, Banach spaces of continuous functions on compact spaces, in: Recent Progress in General Topology (Prague, 2001), M. Hušek (ed.), North-Holland, to appear.

[16] G. Godefroy, N. J. Kalton and G. Lancien, Subspaces of $c_{0}(\mathbb{N})$ and Lipschitz isomorphisms, Geom. Funct. Anal. 10 (2000), 798-820.

[17] W. B. Johnson, J. Lindenstrauss, D. Preiss and G. Schechtman, Almost Fréchet differentiability of Lipschitz mappings between infinite-dimensional Banach spaces, Proc. London Math. Soc. (3) 84 (2002), 711-746.

[18] W. B. Johnson, J. Lindenstrauss and G. Schechtman, Banach spaces determined by their uniform structure, Geom. Funct. Anal. 6 (1996), 430-470.

[19] M. I. Kadec, On complementably universal Banach spaces, Studia Math. 40 (1971), 85-89.

[20] V. Kadets, Lipschitz mappings of metric spaces, Izv. Vyssh. Uchebn. Zaved. Mat. 1985, no. 1, 30-34 (in Russian); English transl.: Soviet Math. (Iz. VUZ) 29 (1985), no. $1,36-41$.

[21] N. J. Kalton, Lipschitz and Hölder-free spaces, to appear.

[22] J. Lindenstrauss, On nonlinear projections in Banach spaces, Michigan Math. J. 11 (1964), 263-287. 
[23] J. Lindenstrauss and D. Preiss, On Fréchet differentiability of Lipschitz maps between Banach spaces, Ann. of Math. 157 (2003), 257-288.

[24] J. Lindenstrauss and L. Tzafriri, Classical Banach Spaces, Vol. 1, Springer, 1977.

[25] G. M. Lövblom, Isometries and almost isometries between spaces of continuous functions, Israel J. Math. 56 (1986), 143-159.

[26] J. Pelant, Embeddings into c $c_{0}$, Topology Appl. 57 (1994), 259-269.

[27] A. Pełczyński, Universal bases, Studia Math. 32 (1969), 247-268.

[28] - Any separable Banach space with the bounded approximation property is a complemented subspace of a space with a basis, ibid. 40 (1971), 239-242.

[29] N. Weaver, Lipschitz Algebras, World Sci., 1999.

Équipe d'Analyse

Université Paris VI

Boîte 186

4, Place Jussieu

75252 Paris Cedex 05, France

E-mail: gig@ccr.jussieu.fr
Department of Mathematics University of Missouri-Columbia Columbia, MO 65211, U.S.A. E-mail: nigel@math.missouri.edu

Received October 3, 2002

Revised version March 14, 2003 